\title{
Clinical sensitivity of prenatal screening for cystic fibrosis via CFTR carrier testing in a United States panethnic population
}

\author{
Glenn E. Palomaki, BS ${ }^{1}$, Stacey C. FitzSimmons, $P h D^{2}$, and James E. Haddow, $M D^{1}$
}

\begin{abstract}
Purpose: To estimate CFTR mutation frequencies, clinical sensitivities (proportions of carrier couples or affected fetuses detected), and birth prevalence estimates for broad racial/ethnic groups and for a panethnic U.S. population. Methods: Published sources of information were identified, corrected when appropriate, and summarized. Combining racial/ethnic-specific mutation frequencies and birth prevalence estimates allowed the computation of panethnic estimates. Results: Two of the 25 recommended mutations do not meet the $0.1 \%$ threshold in a panethnic population set by the American College of Medical Genetics. The clinical sensitivities are estimated to be $71.9 \%, 51.7 \%, 41.6 \%, 88.6 \%$, and $23.4 \%$ for non-Hispanic Caucasians, Hispanic Caucasian, African American, Ashkenazi Jewish Caucasian, and Asian American couples, respectively. Birth prevalence estimates are $1: 2,500,1: 13,500,1: 15,100,1: 2,270$, and 1:35,100, whereas the number of couples needed to screen to detect an affected fetus are about 3,200, 26,120;36,040;2,600, and 129,600, respectively, for the same racial/ethnic groups. Conclusions: Overall, the panethnic estimates for CFTR mutation frequencies are similar to those for non-Hispanic Caucasians. However, large differences in both clinical sensitivity and birth prevalence exist between the broad racial/ethnic groups examined. Whether and how the differences in the numbers of couples needed to screen to detect an affected fetus are to be included in prenatal screening for cystic fibrosis needs to be more explicitly addressed. Genet Med 2004:6(5):405-414.
\end{abstract}

Key Words: cystic fibrosis, CFTR, prenatal screening, clinical sensitivity, ethnicity

The aim of prenatal screening for cystic fibrosis is to identify couples in which both individuals are carriers of CFTR mutations that cause the severe, or classic, forms of cystic fibrosis. If these couples are identified before pregnancy, several reproductive options are available including adoption, artificial insemination, and preimplantation diagnosis. If a carrier couple is identified early in pregnancy, diagnostic testing can be offered, as the fetus has a 1 in 4 chance of being affected. If the fetus has inherited both mutations, the couple can prepare for the delivery or choose to terminate the pregnancy. Whether such screening should be offered to all couples, or to only certain subgroups, is a subject of discussion. One reasonable scenario would be to determine suitability for couples having such testing based on race and ethnicity. The birth prevalence of cystic fibrosis, the proportion of mutations detectable by a given set of CFTR mutations, and the relative frequency of

From the ${ }^{1}$ Foundation for Blood Research, Scarborough, Maine; and ${ }^{2}$ FitzSimmons and Associates: Epidemiology and Clinical Research, Bethesda, Maryland.

Glenn E. Palomaki, BS, 69 US Route 1, PO Box 190, Scarborough, ME.

Additional data, appendixes, and tables are available via the ArticlePlus feature at the Genetics in Medicine Web site, www.geneticsinmedicine.org.

Received: May 3, 2004.

Accepted: June 11, 2004.

DOI: 10.1097/01.GIM.0000139505.06194.39
CFTR mutations have been shown to vary between broadly defined racial/ethnic groups, sometimes by a factor of 10 or more. ${ }^{1}$

In a previous study involving the minimum CFTR mutation panel recommended by the American College of Medical Genetics (ACMG), ${ }^{2}$ we used a variety of published and new sources to derive a composite clinical sensitivity among non-Hispanic Caucasians. ${ }^{3}$ We now extend this analysis to include other racial/ethnic groups appropriate for the United States. Demographic identifiers of race are an important consideration when interpreting certain medical tests, such as the mutation panel being used in prenatal screening for cystic fibrosis. This is also true for ethnicity, a category that describes nonbiological, but socially meaningful, groups. Nearly all studies from the United States use the current Census Bureau racial/ethnic categories, in which race is divided into three broad categories (Caucasian, African American, and Asian American). Additional stratification includes designation of Hispanic/non-Hispanic ethnicity among Caucasians and African Americans. One other important Caucasian ethnicity (Ashkenazi Jewish) is often not collected as part of routine governmental statistics, but can be useful when testing is being done for certain genetic disorders. Although the use of these categories is less than ideal, it nonetheless represents an improvement over 
nonuse for providing practical information for individuals and couples.

The current study aims to identify data sources that document racial/ethnic differences in both the birth prevalence of cystic fibrosis and the distribution of CFTR mutations. The analysis is limited to the 25 mutations included in the recommended minimum panel by the ACMG. ${ }^{2}$ From this information, the clinical sensitivity (proportion of carrier couples or affected fetuses) is computed and compared between groups. An overall panethnic estimate of the mutation frequencies is also derived. This information can be helpful when interpreting prenatal screening results, planning rational screening policies, and determining the impact of prenatal screening for cystic fibrosis on public health.

\section{MATERIALS AND METHODS}

\section{CFTR mutation frequencies}

Criteria for selecting published reports for mutation frequencies are the same as those used in an earlier publication. ${ }^{3}$ Briefly, publications were selected that reported on all (or nearly all) of the recommended panel of 25 mutations, contained results from a relatively large number of clinically affected individuals, had information about race/ethnicity, and were, when possible, from the United States. Data from the International Cystic Fibrosis Consortium were taken from Table 1 of its publication. ${ }^{4}$ Data from the Cystic Fibrosis Foundation National Patient Registry were taken from the year 1999 and stratified according to whether or not the patient was seen

Table 1

CFTR mutation frequencies among Hispanic Caucasians with cystic fibrosis within the recommended minimum testing panel

\begin{tabular}{|c|c|c|c|c|c|}
\hline \multirow[b]{2}{*}{ Order $^{a}$} & \multirow[b]{2}{*}{ Mutation } & \multicolumn{4}{|c|}{ Mutation frequency (\%) } \\
\hline & & CF Consortium $^{b}$ & CF Foundation $^{c}$ & Average & Cumulative \\
\hline 1 & delF508 & 45.51 & 63.25 & 54.38 & 54.38 \\
\hline 2 & G542X & 5.11 & 5.09 & 5.10 & 59.48 \\
\hline 8 & delI507 & 0.59 & 5.02 & 2.81 & 62.29 \\
\hline 22 & R334W & 2.25 & 1.31 & 1.78 & 64.07 \\
\hline 6 & N1303K & 1.65 & 1.67 & 1.66 & 65.73 \\
\hline 10 & $3849+10 \mathrm{kbC}>\mathrm{T}$ & 1.60 & 1.53 & 1.57 & 67.30 \\
\hline 7 & R553X & 0.63 & 0.73 & 0.68 & 67.98 \\
\hline 5 & W1282X & 0.53 & 0.73 & 0.63 & 68.61 \\
\hline 19 & R1162X & 0.57 & 0.58 & 0.58 & 69.19 \\
\hline 3 & G551D & 0.31 & 0.80 & 0.56 & 69.75 \\
\hline 12 & $1717-1 \mathrm{G}>\mathrm{T}$ & 0.10 & 0.44 & 0.27 & 70.02 \\
\hline 4 & $621+1 \mathrm{G}>\mathrm{T}$ & 0.00 & 0.51 & 0.26 & 70.28 \\
\hline 14 & $711+1 \mathrm{G}>\mathrm{T}$ & 0.10 & 0.36 & 0.23 & 70.51 \\
\hline 18 & G85E & 0.10 & 0.36 & 0.23 & 70.74 \\
\hline 11 & $2789+5 G>A$ & 0.10 & 0.22 & 0.16 & 70.90 \\
\hline 13 & $\mathrm{R} 347 \mathrm{P}$ & 0.10 & 0.22 & 0.16 & 71.06 \\
\hline 20 & 2184delA & 0.10 & 0.22 & 0.16 & 71.22 \\
\hline 24 & $3120+1 \mathrm{G}>\mathrm{T}$ & 0.10 & 0.22 & 0.16 & 71.38 \\
\hline 17 & 3569delC & 0.10 & 0.15 & 0.13 & 71.51 \\
\hline 9 & $\mathrm{R} 117 \mathrm{H}$ & 0.00 & 0.22 & 0.11 & 71.62 \\
\hline 23 & $\mathrm{I} 148 \mathrm{~T}$ & 0.10 & 0.07 & 0.09 & 71.71 \\
\hline 25 & 1078delT & 0.10 & 0.07 & 0.09 & 71.80 \\
\hline 16 & $\mathrm{~A} 455 \mathrm{E}$ & 0.10 & 0.00 & 0.05 & 71.85 \\
\hline 21 & $1898+1 \mathrm{G}>\mathrm{A}$ & 0.10 & 0.00 & 0.05 & 71.90 \\
\hline \multirow[t]{2}{*}{15} & $\mathrm{R} 560 \mathrm{~T}$ & 0.00 & 0.00 & 0.00 & 71.90 \\
\hline & All 25 & 59.95 & 83.77 & 71.90 & \\
\hline
\end{tabular}

\footnotetext{
${ }^{a}$ The order is based on that found for non-Hispanic Caucasians. ${ }^{3}$

${ }^{b}$ Based on between 178 and 958 chromosomes (International Cystic Fibrosis Genetic Analysis Consortium. ${ }^{4}$

'Based on 1374 chromosomes from clinically diagnosed persons registered in the Cystic Fibrosis Foundation National Patient Registry.
} 
at one of the eight Cystic Fibrosis Foundation specialized clinical research Therapeutic Development Network clinical care centers.

\section{Racial/ethnic description of pregnancies in the United States}

There are few good sources of race/ethnicity for newborns in the United States. We used the mothers' reported race/ethnicity as a surrogate. Information was obtained from the National Vital Statistics Reports, published by the Centers for Disease Control and Prevention. ${ }^{5}$ Estimates of Ashkenazi Jewish births were derived using information from The National Jewish Population Survey, 2000-01. ${ }^{6}$

\section{Birth prevalence of cystic fibrosis by racial/ethnic group}

This information was obtained from an ACCE report that is available online. ${ }^{7}$ Briefly, the literature was searched for patient registries and newborn screening trials in which full ascertainment of individuals with cystic fibrosis was available. Prenatal screening trials were also utilized. The results for many of the studies needed to be stratified by race/ethnicity or adjusted for length of follow-up, known under-ascertainment, effects of prenatal diagnosis, size of mutation panel, and in one study, a bias introduced during allocation. Consensus estimates and 95\% confidence intervals were computed using a random effects model. Complete description of the methods and results are available in the report. ${ }^{7}$

\section{RESULTS}

\section{CFTR mutation frequencies in Hispanic Caucasians with cystic fibrosis}

Table 1 contains CFTR mutation frequencies among Hispanic Caucasians with clinically defined cystic fibrosis. The second column lists the 25 mutations contained in the ACMG minimum recommended panel. ${ }^{2}$ As a point of reference, the first column numbers the mutation frequencies (from high to low) among non-Hispanic Caucasians affected with cystic fibrosis. ${ }^{3}$ The first data source for mutation frequencies (column 3 ) is derived from published information from the Cystic Fibrosis Consortium. ${ }^{4}$ The present analysis corrects errors in the denominator of the Consortium's data, as described for nonHispanic Caucasians in our earlier publication. ${ }^{3}$ The seven studies selected for analysis include affected individuals from the United States (four studies), Mexico (one study), and South America (two studies). A listing of the original data can be found online (in the ArticlePlus section at http://www. geneticsinmedicine.org). The numbers of chromosomes tested for each mutation range from 178 to 958 . Nearly half (12/25) of the 25 recommended mutations were not tested for in any of the seven studies, and these have been arbitrarily assigned a frequency of 1 per $1000(0.1 \%)$.

The second data source (Table 1, column 4) is an analysis based on the Cystic Fibrosis Foundation National Patient Registry. All individuals in the Cystic Fibrosis Foundation National Patient Registry included in this analysis are from the United States and were reported to be both Caucasian and
Hispanic. It is very likely that they were tested for at least these 25 mutations included in Table 1, if they were seen at a Cystic Fibrosis Foundation-supported specialized clinical research center called the Therapeutic Diagnostic Network (TDN). When the analysis is restricted to individuals from the TDN (the most unbiased set possible), a total of 130 chromosomes is available for analysis. With this small number of observations, it is possible only to obtain reliable estimates for the frequency of delF508 and of unknown/other mutation (other mutations include those mutations that have been identified, but are not included in the recommended 25 mutations). These two frequencies are $60.0 \%$ (95\% CI 51\% to $68 \%$ ) and $20.8 \%$ (95\% CI $14 \%$ to $29 \%$ ), respectively. If all individuals reporting Hispanic Caucasian ethnicity are included, 1374 chromosomes are available. We have chosen to use the larger dataset (Table 1), due to the increased reliability of the frequencies for less common mutations and because the overall rates for this dataset are similar to those found for patients attending the TDN Centers (see ArticlePlus). Only three mutations were never identified (R560T, A455E, and $1898+1 \mathrm{G}>\mathrm{A}$ ). The average of the mutation frequencies from the two data sources is used as the best estimate (column 5). A weighted average is not used, because the differences are unlikely to be due to chance. Rather, they are likely to be caused by limitations or biases inherent in each of the studies which cannot be accounted for by weighting. The last column shows the cumulative frequency.

The frequency of delF508 is about $15 \%$ points higher in the Cystic Fibrosis Foundation National Patient Registry than in the International Cystic Fibrosis Consortium data (63 vs. $46 \%)$. This may be due to under-ascertainment of delF508 in the Consortium's data. ${ }^{3,8}$ The relatively high frequency of delF508 in the Cystic Fibrosis Foundation's data might also be due to the methods of collecting racial/ethnic information. The Cystic Fibrosis Foundation relies on nurse-reported ra$\mathrm{cial} / \mathrm{ethnic}$ information, and it is possible that some fraction of the population had parents or grandparents who were of nonHispanic Caucasian heritage. Such misclassification might overestimate the delF508 mutation frequency. In contrast, several of the studies included in the Consortium's data carefully documented the racial/ethnic origin of both parents. The frequency of delI507 is also higher in the Foundation's data, but there is no obvious explanation. These findings underscore the practical nature of the panethnic mutation panel. The 12 "rare" mutations that were not tested for in the Consortium's data collection were each assigned a frequency of $0.10 \%$, for a total of $1.2 \%$. This is somewhat lower, but consistent with, the observed rate of $2.0 \%$ in the Foundation's data for the same 12 mutations.

The cumulative frequency for the ACMG recommended minimum panel of 25 mutations is about $70 \%$, with a low estimate of $60 \%$ (based on the Consortium's data alone) to a high estimate of $84 \%$ (based on the Cystic Fibrosis Foundation's data alone). This brings into question which of the two estimates should be used in practice. The lower estimate may be best for this racial/ethnic group when it is carefully defined. However, the higher estimate may be more appropriate for 
routine use, in that it reflects the actual performance expected in a population-based screening program that would also rely on self-classification.

\section{CFTR mutation frequencies in African Americans with cystic fibrosis}

Table 2 contains CFTR mutation frequencies among African Americans with clinically defined cystic fibrosis, limited to the 25 CFTR mutations contained in the ACMG minimum recommended panel. ${ }^{2}$ The first data source is again derived from published information from the Cystic Fibrosis Consortium with corrected denominators. ${ }^{3}$ The four studies selected for analysis include only affected individuals from the United States. None of the studies from Africa are included. A listing of the data can be found online (see ArticlePlus). The total numbers of chromosomes tested for each mutation range from 79 to 169 . This relatively small number of tested chromosomes is not unexpected, because cystic fibrosis is less common in African Americans. Only two of the 25 recommended mutations were not tested for in at least one of the studies, and these have been arbitrarily assigned a frequency of $0.1 \%$.

The Cystic Fibrosis Foundation National Patient Registry data are also summarized in Table 2. A preliminary analysis shows that the mutation frequencies among those patients attending TDN clinical care centers are not significantly different from all nurse-reported African Americans in the Cystic Fibrosis National Patient Registry (see ArticlePlus). The larger data-

Table 2

CFTR mutation frequencies among African American individuals with cystic fibrosis within the recommended minimum testing panel

\begin{tabular}{|c|c|c|c|c|c|}
\hline \multirow[b]{2}{*}{ Order $^{a}$} & \multirow[b]{2}{*}{ Mutation } & \multicolumn{4}{|c|}{ Mutation frequency (\%) } \\
\hline & & $\begin{array}{c}\text { CF } \\
\text { Consortium }^{b}\end{array}$ & $\begin{array}{c}\mathrm{CF} \\
\text { Foundation }^{c}\end{array}$ & Average & Cumulative \\
\hline 1 & delF508 & 35.50 & 52.63 & 44.07 & 44.07 \\
\hline 24 & $3120+1 \mathrm{G}>\mathrm{T}$ & 12.50 & 6.64 & 9.57 & 53.64 \\
\hline 8 & delI507 & 0.74 & 3.89 & 2.32 & 55.96 \\
\hline 7 & R553X & 2.37 & 1.37 & 1.87 & 57.83 \\
\hline 2 & G542X & 1.18 & 1.72 & 1.45 & 59.28 \\
\hline 3 & G551D & 0.59 & 1.83 & 1.21 & 60.49 \\
\hline 4 & $621+1 G>T$ & 1.18 & 1.03 & 1.11 & 61.60 \\
\hline 19 & R1162X & 0.74 & 0.57 & 0.66 & 62.26 \\
\hline 22 & $\mathrm{R} 334 \mathrm{~W}$ & 0.74 & 0.23 & 0.49 & 62.75 \\
\hline 12 & $1717-1 \mathrm{G}>\mathrm{T}$ & 0.74 & 0.00 & 0.37 & 63.12 \\
\hline 6 & N1303K & 0.00 & 0.69 & 0.35 & 63.47 \\
\hline 5 & W1282X & 0.00 & 0.47 & 0.24 & 63.71 \\
\hline 10 & $3849+10 \mathrm{kbC}>\mathrm{T}$ & 0.00 & 0.34 & 0.17 & 63.88 \\
\hline 15 & R560T & 0.00 & 0.34 & 0.17 & 64.05 \\
\hline 18 & G85E & 0.00 & 0.23 & 0.12 & 64.17 \\
\hline 9 & $\mathrm{R} 117 \mathrm{H}$ & 0.00 & 0.11 & 0.06 & 64.23 \\
\hline 13 & R347P & 0.00 & 0.11 & 0.06 & 64.29 \\
\hline 17 & 3569delC & 0.00 & 0.11 & 0.06 & 64.35 \\
\hline 21 & $1898+1 G>A$ & 0.00 & 0.11 & 0.06 & 64.41 \\
\hline 20 & 2184delA & 0.10 & 0.00 & 0.05 & 64.46 \\
\hline 23 & $\mathrm{I} 148 \mathrm{~T}$ & 0.10 & 0.00 & 0.05 & 64.51 \\
\hline 11 & $2789+5 G>A$ & 0.00 & 0.00 & 0.00 & 64.51 \\
\hline 14 & $711+1 G>T$ & 0.00 & 0.00 & 0.00 & 64.51 \\
\hline 16 & A455E & 0.00 & 0.00 & 0.00 & 64.51 \\
\hline \multirow[t]{2}{*}{25} & 1078delT & 0.00 & 0.00 & 0.00 & 64.51 \\
\hline & All 25 & 56.46 & 72.42 & 64.51 & \\
\hline
\end{tabular}

\footnotetext{
${ }^{a}$ The order is based on that found for non-Hispanic Caucasians. ${ }^{3}$

${ }^{b}$ Based on between 79 and 169 chromosomes reported by the International Cystic Fibrosis Genetic Analysis Consortium. ${ }^{4}$

'Based on 874 chromosomes from clinically diagnosed persons registered in the Cystic Fibrosis Foundation National Patient Registry.
} 
set that includes all self-declared African Americans has, therefore, been chosen for analysis.

According to both datasets, the frequency of the delF508 mutation is lower in African Americans than in either nonHispanic or Hispanic Caucasians. As in the earlier analysis of Hispanic Caucasians, the Cystic Fibrosis Foundation National Patient Registry has a higher estimated frequency for this mutation $(53 \%)$ than the International Cystic Fibrosis Consortium $(35 \%)$. This is probably due to a higher rate of admixture among the self-declared African Americans in the Cystic Fibrosis Foundation's data, compared to other studies where more extensive data about race/ethnicity were collected. Both datasets agree that $3120+1 \mathrm{G}>\mathrm{T}$ is the second most common mutation among African Americans, but is relatively infrequent among Caucasians. Overall, about $65 \%$ of CFTR mutations among African Americans might be identifiable using the recommended panel. The lower estimate of $56 \%$ might be appropriate when the ethnic background is known to be exclusively African American. The higher estimate of $72 \%$ might be more appropriate, however, in the screening setting where selfdeclared race/ethnicity is relied upon. As before, the average of the results from the two data sources is used as the best estimate.

Other studies in African Americans have been published that overlap or are included in Table 2. In a group of 82 African Americans, one study ${ }^{9}$ reported that the common "Caucasian" mutations accounted for 52 of the 164 affected chromosomes (32\%). The addition of eight more "African American" mutations increased the proportion identified to $75 \%$. One mutation $(3120+1 G>A)$ accounted for more than half of the increase and is included in the recommended panel. One other "African American" mutation (R553X) is also included in that panel. With these two inclusions, the expected proportion of mutations detected is $66 \%$, nearly identical to our estimate of $65 \%$. Another study that has significant overlap with the Cystic Fibrosis Foundation National Patient Registry ${ }^{8}$ reported that the large panels used by the authors (70 or 86 mutations) would be capable of detecting $81 \%$ of African American mutations. The ACMG-recommended panel does not contain several of the mutations detected by this group, and the rate reported in this study (65\%) is reasonably consistent. Overall, these, and several other smaller studies report frequencies that are similar to those reported here, and their inclusion would have little impact on the overall estimates. Although some African Americans/Blacks consider themselves to be Hispanic, this proportion is relatively small. In 2002, 2.3\% of mothers that reported being African American/Black also considered themselves to be Hispanic. ${ }^{5}$ Unfortunately, there are few data available in the literature to make any separate estimates of CFTR mutation frequencies in this group.

\section{CFTR mutation frequencies in Ashkenazi Jewish Caucasians with cystic fibrosis}

Table 3 contains CFTR mutation frequencies among Ashkenazi Jewish Caucasians with clinically defined cystic fibrosis, again limited to the 25 CFTR mutations contained in the
Table 3

CFTR mutation frequencies among Ashkenazi Jewish Caucasian individuals with cystic fibrosis within the recommended minimum testing panel

\begin{tabular}{|c|c|c|c|}
\hline \multirow[b]{2}{*}{ Order $^{a}$} & \multirow[b]{2}{*}{ Mutation } & \multicolumn{2}{|c|}{ Mutation frequency (\%) } \\
\hline & & CF Consortium $^{b}$ & Cumulative \\
\hline 5 & W1282X & 45.92 & 45.92 \\
\hline 1 & delF508 & 31.41 & 77.33 \\
\hline 2 & G542X & 7.55 & 84.88 \\
\hline 10 & $3849+10 \mathrm{kbC}>\mathrm{T}$ & 4.77 & 89.65 \\
\hline 6 & N1303K & 2.78 & 92.43 \\
\hline 12 & $1717-1 \mathrm{G}>\mathrm{T}$ & 0.67 & 93.10 \\
\hline 7 & R553X & 0.22 & 93.32 \\
\hline 3 & G551D & 0.22 & 93.54 \\
\hline 24 & $3120+1 \mathrm{G}>\mathrm{T}$ & 0.10 & 93.64 \\
\hline 21 & $1898+1 \mathrm{G}>\mathrm{A}$ & 0.10 & 93.74 \\
\hline 20 & 2184delA & 0.10 & 93.84 \\
\hline 23 & $\mathrm{I} 148 \mathrm{~T}$ & 0.10 & 93.94 \\
\hline 11 & $2789+5 G>A$ & 0.10 & 94.04 \\
\hline 14 & $711+1 \mathrm{G}>\mathrm{T}$ & 0.10 & 94.14 \\
\hline 8 & delI507 & 0.00 & 94.14 \\
\hline 19 & R1162X & 0.00 & 94.14 \\
\hline 22 & R334W & 0.00 & 94.14 \\
\hline 4 & $621+1 G>T$ & 0.00 & 94.14 \\
\hline 15 & $\mathrm{R} 560 \mathrm{~T}$ & 0.00 & 94.14 \\
\hline 18 & G85E & 0.00 & 94.14 \\
\hline 9 & $\mathrm{R} 117 \mathrm{H}$ & 0.00 & 94.14 \\
\hline 13 & R347P & 0.00 & 94.14 \\
\hline 17 & 3569delC & 0.00 & 94.14 \\
\hline 16 & A455E & 0.00 & 94.14 \\
\hline \multirow[t]{2}{*}{25} & 1078delT & 0.00 & 94.14 \\
\hline & Sum & 94.14 & \\
\hline
\end{tabular}

${ }^{a}$ The order is based on that found for non-Hispanic Caucasians. ${ }^{3}$

${ }^{b}$ Based on between 57 and 503 chromosomes reported by the International Cystic Fibrosis Genetic Analysis Consortium. ${ }^{4}$

ACMG minimum recommended panel. The first data source is again derived from published information from the International Cystic Fibrosis Consortium, ${ }^{4}$ with corrected denominators. ${ }^{3}$ The five studies selected for analysis include four from the United States and one, the largest, from Israel. A listing of the data can be found online (see ArticlePlus). Among these studies, only eight mutations were identified; the most common being W1282X. Overall, about $94 \%$ of mutations could be identified by the recommended panel. The Cystic Fibrosis Foundation National Patient Registry does not contain information about Ashkenazi Jewish heritage and, therefore, is not included in this analysis.

An earlier article ${ }^{10}$ reported that $97 \%$ of mutations were identified in 90 chromosomes from Ashkenazi Jewish individ- 
uals with cystic fibrosis, using a panel of 11 mutations. This is higher than the $94.1 \%$ found in the present analysis, which is based on the larger International Consortium dataset. The data from this smaller study are likely to have been included in the Consortium's data as part of the 500 chromosomes reported from Israel. The higher rate of $97 \%$ has been widely quoted. ${ }^{2} \mathrm{~A}$ more recent study $y^{8}$ reported that $95.4 \%$ of mutations were identified in 24 Ashkenazi Jewish individuals with cystic fibrosis using a panel of 86 mutations, but only $85.4 \%$ were detected using the panel of 70 mutations. Their estimates are based on small numbers but are reasonably consistent with the present estimate of $94.1 \%$, using the smaller recommended minimum panel of 25 mutations. Several studies report that NonAshkenazi Jewish individuals usually have a lower proportion of mutations detected. ${ }^{11-13}$

\section{CFTR mutation frequencies in Asian Americans with cystic fibrosis}

Table 4 contains CFTR mutation frequencies among Asian Americans with clinically defined cystic fibrosis, limited to the 25 CFTR mutations contained in the ACMG minimum recommended panel. ${ }^{2}$ Cystic fibrosis is rare in native Asians and, therefore, there is limited information about mutation frequencies. The Cystic Fibrosis Foundation National Patient Registry contains test results for 66 chromosomes among individuals reporting Asian American heritage, but it is likely that many of these individuals are not entirely of Asian background. In an earlier report using the Cystic Fibrosis National Patient Registry data, ${ }^{14}$ a follow-up inquiry to obtain details of one Cystic Fibrosis Care Center's data revealed that " 4 of the 5 Asians with cystic fibrosis had one Caucasian parent.” In an-

Table 4

CFTR mutation frequencies among Asian American individuals with cystic fibrosis within the recommended minimum testing panel

\begin{tabular}{|c|c|c|c|c|c|}
\hline \multirow[b]{2}{*}{ Order $^{a}$} & \multirow[b]{2}{*}{ Mutation } & \multicolumn{4}{|c|}{ Mutation frequency (\%) } \\
\hline & & Heim et al. ${ }^{b}$ & CF Foundation $^{c}$ & Average & Cumulative \\
\hline 1 & delF508 & 18.80 & 59.09 & 38.95 & 38.95 \\
\hline 10 & $3849+10 \mathrm{kbC}>\mathrm{T}$ & 0.00 & 10.61 & 5.31 & 44.26 \\
\hline 3 & G551D & 6.30 & 0.00 & 3.15 & 47.41 \\
\hline 6 & N1303K & 0.00 & 1.52 & 0.76 & 48.17 \\
\hline 8 & delI507 & 0.00 & 1.52 & 0.76 & 48.93 \\
\hline 2 & G542X & 0.00 & 0.00 & 0.00 & 48.93 \\
\hline 4 & $621+1 G>T$ & 0.00 & 0.00 & 0.00 & 48.93 \\
\hline 5 & W1282X & 0.00 & 0.00 & 0.00 & 48.93 \\
\hline 7 & $\mathrm{R} 553 \mathrm{X}$ & 0.00 & 0.00 & 0.00 & 48.93 \\
\hline 9 & $\mathrm{R} 117 \mathrm{H}$ & 0.00 & 0.00 & 0.00 & 48.93 \\
\hline 11 & $2789+5 G>A$ & 0.00 & 0.00 & 0.00 & 48.93 \\
\hline 12 & $1717-1 \mathrm{G}>\mathrm{T}$ & 0.00 & 0.00 & 0.00 & 48.93 \\
\hline 13 & $\mathrm{R} 347 \mathrm{P}$ & 0.00 & 0.00 & 0.00 & 48.93 \\
\hline 14 & $711+1 \mathrm{G}>\mathrm{T}$ & 0.00 & 0.00 & 0.00 & 48.93 \\
\hline 15 & R560T & 0.00 & 0.00 & 0.00 & 48.93 \\
\hline 16 & $\mathrm{~A} 455 \mathrm{E}$ & 0.00 & 0.00 & 0.00 & 48.93 \\
\hline 17 & 3569delC & 0.00 & 0.00 & 0.00 & 48.93 \\
\hline 18 & G85E & 0.00 & 0.00 & 0.00 & 48.93 \\
\hline 19 & R1162X & 0.00 & 0.00 & 0.00 & 48.93 \\
\hline 20 & 2184delA & 0.00 & 0.00 & 0.00 & 48.93 \\
\hline 21 & $1898+1 G>A$ & 0.00 & 0.00 & 0.00 & 48.93 \\
\hline 22 & R334W & 0.00 & 0.00 & 0.00 & 48.93 \\
\hline 23 & $\mathrm{I} 148 \mathrm{~T}$ & 0.00 & 0.00 & 0.00 & 48.93 \\
\hline 24 & $3120+1 \mathrm{G}>\mathrm{T}$ & 0.00 & 0.00 & 0.00 & 48.93 \\
\hline \multirow[t]{2}{*}{25} & 1078delT & 0.00 & 0.00 & 0.00 & 48.93 \\
\hline & Sum & 25.10 & 72.74 & 48.93 & \\
\hline
\end{tabular}

\footnotetext{
${ }^{a}$ The order is based on that found for non-Hispanic Caucasians. ${ }^{3}$

${ }^{b}$ Based on 20 chromosomes.

${ }^{c}$ Based on 66 chromosomes from clinically diagnosed persons registered in the Cystic Fibrosis Foundation National Patient Registry.
} 
other study of three Asians with cystic fibrosis, ${ }^{15}$ one had an American Caucasian father and one of the remaining two cases was a product of a consanguineous relationship. In one of the larger studies involving 10 Asian individuals with cystic fibrosis, ${ }^{8}$ only about one-quarter of the mutations were identified by the panel of 86 . It is not clear whether careful examination of ethnic heritage was undertaken or whether race was self-declared. In an extensive review of the literature in 1998, 14 genotyped Asian individuals with clinically diagnosed cystic fibrosis were identified. Among the three individuals where one copy of the delF508 mutation was found, Caucasian admixture was documented. ${ }^{16}$ Thus, there is no documented example of the delF508 mutations occurring in native Asians.

Although a confident estimate for the proportion of mutations detected among Asian Americans is not possible, two findings are clear. An important proportion of self-reported Asian individuals in the United States with cystic fibrosis will likely be a product of Asian American and non-Asian American parents. The overall proportion of mutations detected is likely to be lower for this racial/ethnic group than for any of the other studies so far, but higher than that found among Asians in their native lands.

\section{Clinical sensitivity for selected racial/ethnic groups}

The clinical sensitivity of CFTR mutation testing can be defined as the proportion of individuals clinically diagnosed with "classic" cystic fibrosis that has two mutations identified. In the present context, the clinical sensitivity is limited by the proportion that can be diagnosed using the 25 mutations in the ACMG recommended minimum panel. This definition does not consider the advantages and disadvantages of various screening models (e.g., sequential or couple) by which a carrier couple might be identified, ${ }^{17}$ because, in theory, they have equivalent clinical sensitivity. More complex issues, such as the usefulness of extended panel testing for partners of known carrier individuals, are beyond the scope of this report.

Under the Hardy-Weinburg assumption, the clinical sensitivity will be the square of the known proportion of identifiable mutations. This computation also assumes that each mutation alone, or in combination with another of the 25 mutations, will cause classic cystic fibrosis in the offspring. For example, among Hispanic Caucasians couples, $71.9 \%$ of classic cystic fibrosis mutations are identifiable (Table 1). The corresponding clinical sensitivity is $51.7 \%(71.9 \times 71.9)$. Thus, just over half of Hispanic Caucasian carrier couples could be identified using the proposed 25 mutation panel. By extension, $51.7 \%$ of fetuses with two identifiable mutations could also be detected. Similarly, the clinical sensitivities for African American, Ashkenazi Jewish Caucasian, and Asian American couples are $41.6 \%, 88.6 \%$, and $23.9 \%$, respectively. Figure 1 graphically displays how the clinical sensitivity for each of these groups increases, as the number of mutations being tested increases. For each group, CFTR mutations are added in order of their frequencies within that group.

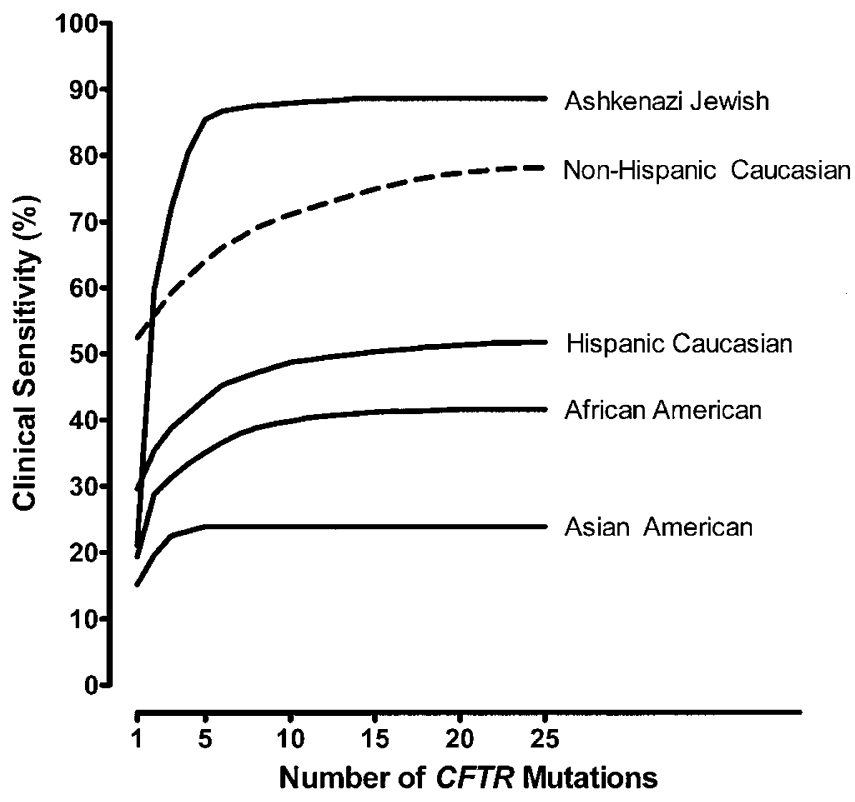

Fig. 1. Clinical sensitivity for detecting cystic fibrosis, using a panel of 25 CFTR mutations in five racial/ethnic groups. Clinical sensitivity (the square of the proportion of detected disease-causing mutations) is the proportion of carrier couples or affected fetuses that can be identified and is shown on the vertical axis. Horizontal axis shows the American College of Medical Genetics recommended panel of 25 mutations. For each group, the mutations are added in order from most to least common within that group. The clinical sensitivity reported is considered a maximum, as analytic sensitivity is assumed to be $100 \%$.

\section{Birth prevalence of classic cystic fibrosis by racial/ethnic group}

In order to compute the CFTR mutation frequencies in a panethnic population, it is necessary to have an estimate of cystic fibrosis incidence (or birth prevalence) for each racial/ ethnic group. One set of estimates is available on the Centers for Disease Control and Prevention web site as part of a review of prenatal cystic fibrosis screening. ${ }^{7}$ That analysis identifies three types of studies that can be used to estimate the birth prevalence of classic cystic fibrosis: closed population cohorts that rely on the clinical definition of cystic fibrosis; newborn screening programs for cystic fibrosis with complete ascertainment; and prenatal screening trials that can determine mutation frequencies using specified testing panels. The resulting birth prevalence estimates for the five racial/ethnic groups are shown in Table 5.

\section{Definition of a panethnic population for the United States}

The United States government routinely collects self-declared maternal racial/ethnic information as part of state birth records. The categories can be stratified into non-Hispanic Caucasian, Hispanic Caucasian, African American, Asian American, and American Indian. The proportion of the population that is Ashkenazi Jewish is derived using two estimates: 5 million of the US population of 293 million (1.7\%) is reported to be Jewish, and the fertility rate for Jewish women is lower than that of the general population. ${ }^{6}$ From this, an estimated 60,000 (1.5\%) Ashkenazi Jewish births occur, and these have been subtracted from the non-Hispanic Caucasian birth 
Table 5

Estimated birth prevalence of classic cystic fibrosis for five broad racial/ethnic groups

\begin{tabular}{llcl}
\hline Race/Ethnicity & $\begin{array}{c}\text { Study } \\
\text { type }\end{array}$ & $\begin{array}{c}\text { No. of } \\
\text { reports }\end{array}$ & $\begin{array}{c}\text { Birth Prevalence (1:n) } \\
(95 \% \text { confidence interval })\end{array}$ \\
\hline Non-Hispanic Caucasian & Registries & 3 & $2,500(2,370$ to 2,630$)$ \\
& Newborn & 17 & $2,510(2,290$ to 2,750$)$ \\
& Prenatal & 13 & $2,490(2,220$ to 2,780$)$ \\
Consensus & 33 & $2,500(2,380$ to 2,630$)$ \\
Hispanic Caucasian & Registries & 1 & $9,200(8,050$ to 10,600$)$ \\
& Prenatal & 3 & $16,670(9,090$ to 33,330$)$ \\
African American & Consensus & 4 & $13,500(6,800$ to 27,000$)$ \\
Ashkenazi Jewish & Registries & 3 & $15,100(14,800$ to 15,300$)$ \\
& Registries & 1 & $3,120(2,411$ to 4,063$)$ \\
Asian American & Prenatal & 4 & $2,040(1,695$ to 2,500$)$ \\
\hline
\end{tabular}

group. Table 6 summarizes the proportion of mothers in the United States population assigned to each of these five racial/ ethnic groups. The American Indian group is included for completeness but is not included in the present analysis.

\section{Clinical sensitivity for prenatal cystic fibrosis screening in a United States panethnic population}

Table 7 shows the cumulative proportion of CFTR mutations detected using the recommended mutation panel in a United States panethnic population. The first step in determining the clinical sensitivity is to determine the number of CFTR mutation carriers for each mutation in each of the racial/ ethnic groups. As an example, consider computing the number of Hispanic Caucasians expected to carry the delF508 mutation. Among the 877,000 Hispanic Caucasian births (Table 6), 15,094 mothers are expected to be carriers of a CFTR mutation

Table 6

Self-reported maternal race/ethnicity for U.S. births during 2002

\begin{tabular}{lcr}
\hline \multirow{2}{*}{\begin{tabular}{lcr} 
Gaternal Racial/Ethnic \\
\cline { 2 - 3 }
\end{tabular}} & \multicolumn{2}{c}{ U.S. births in 2002} \\
\hline Non-Hispanic Caucasian & 21.8 & No. $^{a}$ \\
Hispanic Caucasian $^{\mathrm{b}}$ & 14.8 & $2,238,000$ \\
African American & 5.3 & 877,000 \\
Asian American & 1.5 & 594,000 \\
Ashkenazi Jewish Caucasianc & 1.0 & 211,000 \\
American Indian & 100 & 60,000 \\
All & & $4,022,000$ \\
\hline
\end{tabular}

${ }^{a}$ Numbers rounded to the nearest 1,000 .

${ }^{b}$ The 16,000 reported Hispanic Blacks were included in the category African American.

'Not reported on birth certificates-method of estimation described in the text.
$(877,000 / 58.1$ from Table 5), and 8,207 of these will have the delF508 mutation $(15,094 \times 0.5438$ from Table 1$)$. In only two instances (indicated by bolded numbers) is the number of carriers identified for a specific mutation in the non-Hispanic Caucasian group exceeded by another racial/ethnic group. Hispanic Caucasians have a higher number of R334W mutations, and African Americans have a higher number of $3120+1 \mathrm{G}>$ T mutations. Overall, 99,684 carriers can be identified in this population, using the recommended minimum panel of 25 mutations, assuming that no analytic errors occur. These carriers represent $84 \%$ of all CFTR mutation carriers in the entire population. This indicates that the clinical sensitivity is $71 \%(0.84 \times 0.84)$ for carrier couples (and affected fetuses), using the recommended panel. Although non-Hispanic Caucasians and Ashkenazi Jewish Caucasians represent about 57\% of the population, these two groups contain $82 \%$ of the CFTR carrier individuals detectable by the panel of 25 mutations. Overall, the mutation frequencies in the panethnic population are similar to those in the non-Hispanic Caucasian population.

\section{DISCUSSION}

Both selected CFTR mutation frequencies and the total proportion of mutations identified vary significantly between the five racial/ethnic groups examined in this study, along with a previous study of non-Hispanic Caucasians. ${ }^{3}$ In addition, the prevalence of cystic fibrosis in these groups varies by more than a factor of 10. Such differences are large enough to deserve being taken into account by policy-makers and health care providers, even though such information may not always be reliable or easily collected. An interesting finding is that two mutations originally included in the ACMG minimum recommended panel (I148T and 1078delT) fall below the $0.1 \%$ level in a panethnic population initially chosen as a lower limit for inclusion. Other problems associated with testing for 
Table 7

Estimated number of carriers of the 25 recommended CFTR mutations by racial/ethnic group and weighted average, representing the panethnic population in the United States for 2002

\begin{tabular}{|c|c|c|c|c|c|c|c|c|}
\hline \multirow[b]{2}{*}{ Order } & \multirow[b]{2}{*}{ CFTR mutation } & \multicolumn{6}{|c|}{ Number of CFTR Mutation Carriers } & \multirow[b]{2}{*}{$\begin{array}{c}\text { Panethnic } \\
\text { frequency, \% }\end{array}$} \\
\hline & & $\begin{array}{l}\text { Non-Hispanic } \\
\text { Caucasian }\end{array}$ & $\begin{array}{l}\text { Hispanic } \\
\text { Caucasian }\end{array}$ & $\begin{array}{l}\text { African } \\
\text { American }\end{array}$ & $\begin{array}{c}\text { Asian } \\
\text { American }\end{array}$ & $\begin{array}{l}\text { Ashkenazi } \\
\text { Jewish }\end{array}$ & Total & \\
\hline 1 & delF508 & 64,779 & 8,207 & 4,272 & 886 & 796 & 78,940 & 66.31 \\
\hline 2 & G542X & 2,039 & 770 & 141 & 0 & 191 & 3,141 & 2.64 \\
\hline 5 & W1282X & 1,342 & 95 & 23 & 0 & 1,164 & 2,624 & 2.20 \\
\hline 3 & G551D & 2,013 & 85 & 117 & 72 & 6 & 2,293 & 1.93 \\
\hline 4 & $621+1 G>T$ & 1,404 & 39 & 108 & 0 & 0 & 1,551 & 1.30 \\
\hline 6 & N1303K & 1,136 & 251 & 34 & 17 & 70 & 1,508 & 1.27 \\
\hline 7 & $\mathrm{R} 553 \mathrm{X}$ & 778 & 424 & 225 & 17 & 0 & 1,444 & 1.21 \\
\hline 8 & delI507 & 787 & 103 & 181 & 0 & 6 & 1,077 & 0.90 \\
\hline 10 & $3849+10 \mathrm{kbC}>\mathrm{T}$ & 519 & 237 & 16 & 121 & 121 & 1,014 & 0.85 \\
\hline 24 & $3120+1 G>T$ & 72 & 24 & 928 & 0 & 3 & 1,027 & 0.86 \\
\hline 9 & $\mathrm{R} 117 \mathrm{H}$ & 626 & 17 & 6 & 0 & 0 & 649 & 0.55 \\
\hline 12 & $1717-1 \mathrm{G}>\mathrm{T}$ & 429 & 41 & 36 & 0 & 17 & 523 & 0.44 \\
\hline 11 & $2789+5 G>A$ & 429 & 24 & 0 & 0 & 3 & 456 & 0.38 \\
\hline 13 & R347P & 403 & 24 & 6 & 0 & 0 & 433 & 0.36 \\
\hline 14 & $711+1 \mathrm{G}>\mathrm{T}$ & 385 & 35 & 0 & 0 & 3 & 423 & 0.36 \\
\hline 22 & R334W & 125 & 269 & 47 & 0 & 0 & 441 & 0.37 \\
\hline 15 & $\mathrm{R} 560 \mathrm{~T}$ & 340 & 0 & 16 & 0 & 0 & 356 & 0.30 \\
\hline 19 & R1162X & 206 & 88 & 64 & 0 & 0 & 358 & 0.30 \\
\hline 17 & 3569delC & 304 & 20 & 6 & 0 & 0 & 330 & 0.28 \\
\hline 16 & $\mathrm{~A} 455 \mathrm{E}$ & 304 & 8 & 0 & 0 & 0 & 312 & 0.26 \\
\hline 18 & G85E & 259 & 35 & 12 & 0 & 0 & 306 & 0.26 \\
\hline 20 & 2184delA & 152 & 24 & 5 & 0 & 3 & 184 & 0.15 \\
\hline 21 & $1898+1 G>A$ & 143 & 8 & 6 & 0 & 3 & 160 & 0.13 \\
\hline 23 & $\mathrm{I} 148 \mathrm{~T}$ & 80 & 14 & 5 & 0 & 3 & 102 & 0.09 \\
\hline \multirow[t]{2}{*}{25} & 1078delT & 18 & 14 & 0 & 0 & 0 & 32 & 0.03 \\
\hline & All & 79,072 & 10,856 & 6,193 & 1,113 & 2,389 & 99,684 & 84.00 \\
\hline
\end{tabular}

Bolded numbers indicate mutations that are more likely to be found in a racial/ethnic group other than non-Hispanic Caucasians.

the I148T mutation/polymorphism have been discussed elsewhere. ${ }^{18}$

All of the results provided in the current study assume that each individual belongs to a single racial/ethnic group and that both of the partners belong to the same racial/ethnic group. These assumptions will have little or no impact on the estimates of panethnic CFTR mutation frequencies, as these frequencies are dominated by the contributions made by the non-Hispanic Caucasian population. Specific estimates for proportions of mutations identified and carrier couples detected could be made for mixed racial/ethnic populations, but those computations are beyond the scope of this study.

It is unlikely that newly found mutations would be of a high enough frequency among a large enough racial/ethnic group to cause the panethnic frequency of that mutation to be above the $0.1 \%$ threshold. In order for the panethnic frequency to be above the $0.1 \%$ cutoff level, a mutation only found among one of the racial/ethnic groups would need to represent $0.13 \%$ of disease causing mutations among non-Hispanic Caucasians. However, a mutation would need to represent $0.9 \%$ of all CFTR mutations among non-Hispanic Caucasians, 1.2\% among African Americans, 2.9\% of Ashkenazi Jewish Caucasians, or $4.9 \%$ of Asian Americans, in order for the panethnic frequency to be at least $0.1 \%$.

When policy makers consider whether or not to recommend adding a CFTR mutation to the existing panel, it is important to determine the increase in clinical sensitivity, not just the increase in the proportion of mutations identified. For exam- 
ple, a 1.0\% increase in the proportion of mutations identified among Ashkenazi Jewish Caucasians (from 94.14\% to 95.14\%; Table 3) would result in a $1.89 \%$ point increase in clinical sensitivity (from $88.62 \%$ to $90.51 \%$ ). However, that same increase in the proportion of mutations identified among Asian Americans (from 48.93 to 49.93 ; Table 4 ) would result in only a $0.99 \%$ point increase in clinical sensitivity (23.94\% to $24.83 \%$ ).

The number of pregnancies needed to be screened to identify a carrier couple is another way to present the variations by race/ethnicity in cystic fibrosis birth prevalence and proportion of mutations identified by the recommended panel. In other words, for each racial/ethnic group, how many couples would need to be screened in order to identify a carrier couple (or an affected fetus)? These numbers allow policy makers to more clearly view the resources necessary to offer prenatal screening services. Among non-Hispanic Caucasians, one carrier couple can be identified for every 800 couples screened and one affected fetus detected for every 3,200 couples screened (similar estimates of 650 couples screened per carrier couple and 2,600 couples screened per affected fetus are found for Ashkenazi Jewish Caucasians). Those same rates are about 10 times higher for Hispanic Caucasians at 6,530 couples screened per carrier couple and 26,120 couples screened per affected fetus detected. Among African American and Asian American couples, the estimates are even higher, at 9,010 and 36,040 couples, and 32,400 and 129,600 couples, respectively. These numbers clearly delineate the diminishing returns found when offering screening in some racial/ethnic groups.

The recommendations made by the American College of Medical Genetics and American College of Obstetricians and Gynecologists ${ }^{1}$ do contain a subtle but important distinction with regards to prenatal screening for cystic fibrosis by race/ ethnicity: ". . . screening should be offered to those at higher risk of having children with CF (Caucasians and Ashkenazi Jews) and in whom the testing is most sensitive...." This recommendation is clearly based on the higher prevalence and higher clinical sensitivity in these groups and suggests that prenatal care providers should be actively offering such screening to pregnant or prepregnant couples in these two groups. Hispanic Caucasians are specifically referred to as a separate group and appear not to be included in this recommendation. The recommendations continue by adding that "screening be made available to couples in other racial and ethnic groups who are at lower risk and in whom the test may be less sensitive." The reasons why a distinction between these two groups has been made is clear, but the difference between "offered" and "made available" is not. This appears to suggest that pregnant or prepregnant couples could be screened if they expressed an interest, but would not be actively approached. This distinction may be too subtle for routine practice; clearer guidance for health care providers and laboratories would be helpful.

Our analysis provides estimates of frequencies for selected CFTR mutations in a panethnic U.S. population. These esti- mates are based on the best available information at this time for each of five racial/ethnic groups. The findings suggest that two of the 25 mutations on the ACMG recommended panel do not reach the level of $0.1 \%$ in the panethnic population. In addition, we have provided the proportion of mutations identified, clinical sensitivity, numbers needed to screen to identify a carrier couple (or affected fetus), and birth prevalence for five racial/ethnic groups. This information can be of use to health providers, screening laboratories, and policy makers.

\section{ACKNOWLEDGMENTS}

This work was performed as part of an ACCE Review, and support was provided by a cooperative agreement (UR3/ CCU319352) with the Centers for Disease Control and Prevention, Office of Genomics and Disease Prevention.

\section{References}

1. American College of Obstetricians and Gynecologists, American College of Medical Genetics. Preconception and prenatal carrier screening for cystic fibrosis. Clinical and laboratory guidelines. Washington, DC, 2001.

2. Grody WW, Cutting GR, Klinger KW, Richards CS, Watson MS, Desnick RJ. Laboratory standards and guidelines for population-based cystic fibrosis carrier screening. Genet Med 2001;3:149-154

3. Palomaki GE, Haddow JE, Bradley LS, FitzSimmons SC. Updated assessment of cystic fibrosis mutation frequencies in non-Hispanic Caucasians. Genet Med 2001;4:90-94.

4. Kazazian HH for the Cystic Fibrosis Genetic Analysis consortium. Population variation of common cystic fibrosis mutations. Hum Mutat 1994;4:167-177.

5. Martin JA. Hamilton BE, Sutton PD, Ventura SJ, Menacker F, Munson ML. Births: final data for 2002. National Vital Statistics Report, DHHS no. (204-1120) 2003;52.

6. The National Jewish Population Survey 2000-01: Strength, Challenge and Diversity in the American Jewish Population. 2003, United Jewish Communities, New York.

7. Centers for Disease Control and Prevention. Available at: http://www.cdc. gov.genomics/activities/fbr.htm. Accessed May 2004.

8. Heim RA, Sugarman EA, Allitto BA. Improved detection of cystic fibrosis mutations in the heterogeneous US population using an expanded, pan-ethnic mutation panel. Genet Med 2001;3:168-176.

9. Macek M, Mackova A, Hamosh A et al. Identification of common cystic fibrosis mutations in African-Americans with cystic fibrosis increases the detection rate to 75\%. Am J Hum Genet 1997;60:1122-1127.

10. Abeliovich D, Lavon IP, Lerer I, Cohen T, Springer C, Avital A et al. Screening for five mutations detects $97 \%$ of cystic fibrosis (CF) chromosomes and predicts a carrier frequency of 1:29 in the Jewish Ashkenazi population. Am J Hum Genet 1992;51:951-956.

11. Kerem B, Chiba-Falek O, Kerem E. Cystic fibrosis in Jews: Frequency and mutation distribution. Genet Test 1997;1:35-39.

12. Kerem E, Kalman YM, Yahav Y et al. Highly variable incidence of cystic fibrosis and different mutation distribution among different Jewish ethnic groups in Israel. Hum Genet 1995;96:193-197.

13. Orgad S, Neumann S, Loewenthal R, Netanelov-Shapira I, Gazit E. Prevalence of cystic fibrosis mutations in Israeli Jews. Genet Test 2001;5:47-52.

14. Hamosh A, FitzSimmons SC, Macek M, Knowles MR, Rosenstein BJ, Cutting GR. Comparison of the clinical manifestations of cystic fibrosis in black and white patients. J Pediatr 1998;132:255-259.

15. Macek M, Mercier B, Mackova A, Miller PW, Hamosh A, Ferec C et al. Sensitivity of the denaturing gradient gel electrophoresis technique in detection of known mutations and novel Asian mutations in the CFTR gene. Hum Mutat 1997;9:136-147.

16. Suwanjutha S, Huang NN, Wattanasirichaigoon D, Sura T, Harris A, Macek M. Case report of a Thai male cystic fibrosis patient with the $1898+1 \mathrm{G}>\mathrm{T}$ splicing mutation in the CFTR gene: A review of east Asian cases. Hum Mutat 1998;12:361.

17. Wald NJ, Morris JK, Rodeck CH, Haddow JE, Palomaki GE. Cystic fibrosis: selecting the prenatal screening strategy of choice. Prenat Diagn 2003;23:474-483.

18. Rohlfs EM, Zhou Z, Sugarman EA, Heim RA, Pace RG, Knowles MR et al. The I148T CFTR allele occurs on multiple haplotypes: a complex allele is associated with cystic fibrosis. Genet Med 2002;4:319-323. 